\title{
A KNOWLEDGE SUPPORTING SYSTEM FOR DATA ANALYSIS
}

\author{
Hiroyuki Minami*, Masahiro Mizuta ${ }^{\dagger}$ and Yoshiharu Sato ${ }^{\dagger}$
}

\begin{abstract}
Most data analysis systems seem to be convenient for statisticians, but novices in statistics cannot master them easily since it is hard to select appropriate procedures for an observation. The application with the technique of knowledge processing to data analysis is an attractive idea, but there is little research on selecting procedures because of some difficulties in the construction of its knowledge. We shall discuss its difficulties and how to construct the knowledge of data analysis, and offer an implementation of the system with knowledge supports.
\end{abstract}

\section{Introduction}

A computer is an indispensable tool for data analysis, and due to the progress in computer science, the usage has been improved. For example, it plays an important role in graphic display and user interface. S-Language shown in Becker etal.(1988) and SAS-software provide the higher graphical representation and operation. Novices in statistics as well as statistical experts can operate the systems easily, but even if the operation is easy, most novices could not select or judge appropriate procedures because they have little knowledge about statistics.

Some research on this problem, supplemented with a function of techniques in knowledge engineering to data analysis systems, have been reported. Most of them, however, are intended for a specific method or specific area. Few applications have been reported for generic data analysis systems.

In this paper, we introduce a data analysis system which has a supporting function which suggests appropriate statistical procedures based on the observation, the purpose of the analysis and the knowledge bases. This system is written with S-Language (in the data analysis part), DEC-10 Prolog (in the knowledge processing part), and C (other details).

\section{Data analysis and knowledge processing}

There are two aspects in considering a relation between data analysis and knowledge processing. One is a case in which a data analysis applies to knowledge processing, for example,

\footnotetext{
*Doctoral Program, Graduate School of Information Engineering, Faculty of Engineering, Hokkaido

University, Sapporo 060, Japan

tDivision of Information and Graphics Science, Faculty of Engineering, Hokkaido University, Sapporo 060, Japan

Key words: Data analysis system, Expert system, Knowledge processing
} 
the application of Bayes probability theory or Shafer-Dempster theory to decision making (Some applications are explained in Furukawa and Mizoguchi(eds.)(1988) and Ishizuka and Kobayashi(eds.)(1991)). The other is to do data analysis itself more easily using the techniques of knowledge processing. Our system belongs to the latter.

There are many investigations of data analysis systems applied to knowledge processing, but these are restricted to some specific methods for its application. Gale(1986)'s REX, RASS developed by Nakano etal.(1991) are based on regression analysis. The former aims at CAI(Computer Assisted Instruction) and the latter constructs an object-oriented system. In contrast with it, there are few for selecting or judging appropriate procedures according to the user's purpose. Its possibility has been shown in DINDE developed by Oldford and Peters(1988).

Regression analysis is not the only method to which we can apply knowledge processing, so we shall try to apply knowledge processing techniques to many other procedures.

There are two reasons why there is little research on a system for selecting appropriate procedures with knowledge support.

1. Data analysis provides analytical methods for one's subject, thus we usually use the knowledge in the specific field in question. To have a large amount of wide knowledge, however, we must make the same effort as we build a problem solver or expert system for a domain the data belongs to. So, it is difficult to construct a generic analysis system for several domains.

2. Mainly, in knowledge processing, the knowledge is represented by symbolic information (qualitative). Since data analysis treats quantitative information, it is hard to define and construct qualitative knowledge of data analysis.

We shall discuss these two difficulties from the following viewpoints.

On 1, to treat meta-knowledge on data (ex. a unit of measurement, etc.) as knowledge elements will assist a judgment of procedures formally.

In terms of 2, as we tried previously in Minami and Mizuta(1990), we can derive adequate procedures qualitatively from the features of data. Thus, the qualitative knowledge is considered to be a tool for selection of procedures.

Now, in spite of some remaining difficulties, it seems that the application of knowledge processing techniques to data analysis is quite significant.

\section{A formulation of data analysis}

We shall consider how to construct a design of data analysis by qualitative information. A data analysis may be considered to include the following procedures:

Step 1 Decide a main procedure based on the purpose and the observations.

Step 2 When the main procedure cannot deal with the observations directly, choose a procedure or procedures for transforming the observations to get adequate input suitable for the main procedure if possible.

Step 3 Choose a procedure or procedures which display or output the results. 


\section{A Knowledge Supporting System for Data Analysis}

Step 4 When the results of the main procedure do not satisfy the input condition of the procedure(s) chosen in Step 3, choose a different procedure or procedures to transform them adequately if possible.

Step 1 and Step 3 are realized with a simple search which is used in retrieving databases, deterministically in other words. In Step 2 and Step 4, however, we must search them non-deterministically.

We can use the technique of "problem solving" which is a basic approach in artificial intelligence (AI). This is to get a queue of operators which accomplishes a transition from an initial status to a goal status. An operator has three conditions. The operator can be selected when the current status satisfies its Precondition. When the operator applies to the current status, some information provided as Delete list are canceled and some as Add list are added to the status.

We use this principle directly, that is, treat a procedure as an operator, the information of input data as an initial status, and the information of the data which is suitable for a next procedure as a goal status.

Now, we must collect some knowledge not only for a simple search in Step 1 and Step 3 but also for problem solving in Step 2 and Step 4.

\section{Implementation of knowledge bases}

We shall define some terminology:

\section{Procedure}

Each method included in data analysis techniques. To distinguish it from others, we call the main procedure Core Procedure.

\section{Data Information}

Qualitative information of the data which are input for a procedure. "Data Information" consists of structures, scales, dimensions, rows, columns, and so on.

\section{Purpose}

The purpose of data analysis. The purposes are given by various expressions, for instance, sometimes notation or symbol defined for the procedure (PCA, etc.), sometimes ambiguous motions (Classification, etc.).

We have discussed the knowledge base configuration about data analysis in Minami et al.(1991) and Minami and Mizuta(1992). Since all of them treat a scale of data as the least common data information, we can only deal with the procedures of scale transformation. In addition, the relation between "Purpose" and the other information was not clear in our knowledge base.

Here, we discuss the knowledge base as follows: (Both kinds of knowledge mentioned below are used after interpreted to Prolog.)

1. Knowledge for "Core Procedure"

It is used to get "Core Procedure" for the purpose appropriately. The format and example are shown in Figure 1. 
The "Conditions" in Figure 1 involves "Data Information" of the initial observations which cannot be described in knowledge for "Procedure" but is available for selecting "Core Procedure" The elements of the knowledge consist of the external criteria and the scales of data.

The matching of "Core Procedure" and "Purpose" (and "Data Information" of the observation, partly) is not one to one. We cannot always get "Core Procedure" uniquely because there will be many candidates of "Core Procedure" satisfying the retrieval information.

The example shows that the purpose "Classification" matches the procedure "Cluster Analysis" if the "Data Information" of the observation doesn't have external criteria. For the observation from two groups, it matches "Discriminant Analysis" if all the scales of the observation are interval, or "Quantification II" if not.

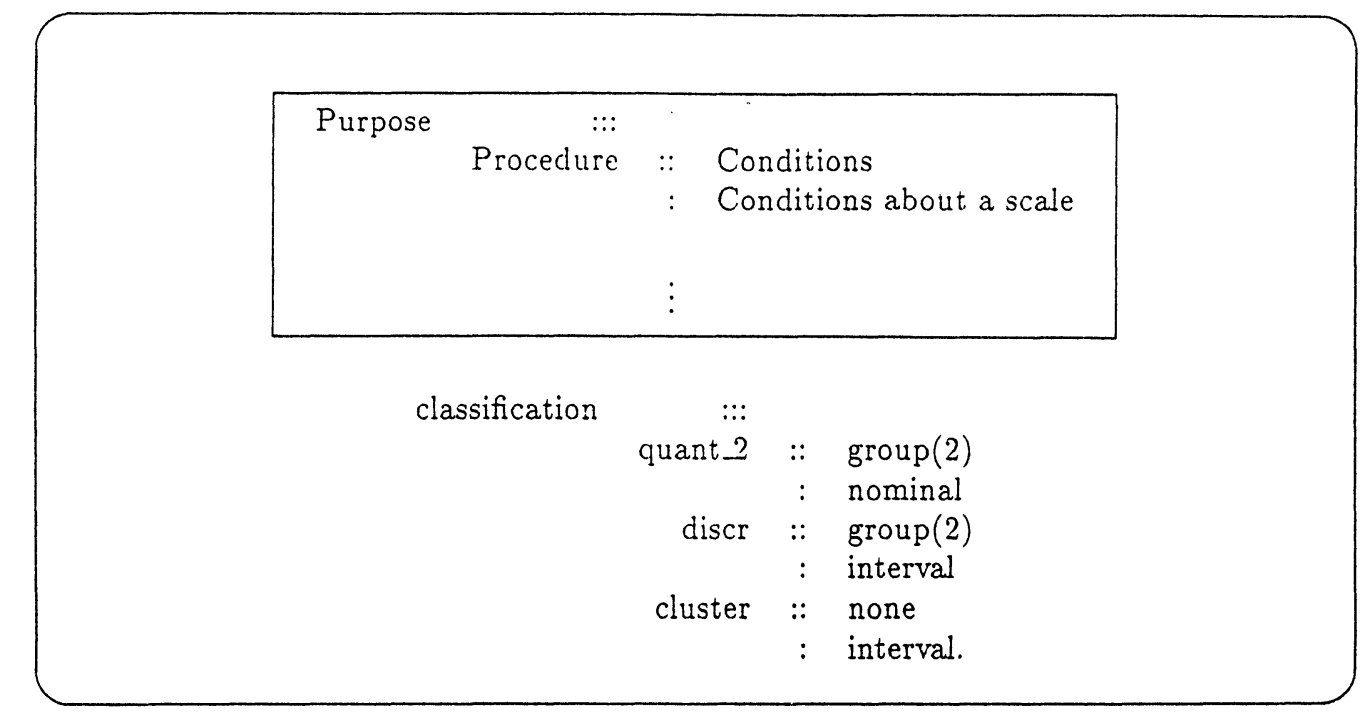

Figure 1: An example of knowledge base for "Core Procedure" (Classification)

2. Knowledge for construction of "Procedure" queues

Each of them consists of preconditions (see below why they are plural), delete-list and add-list, and the "Options". The "Options" are the knowledge required in executing the procedure. It consists of the parameters and default values of the procedure, and the quantitative conditions judged in execution. A format and example are shown in Figure 2.

This knowledge base has two preconditions. "Best precondition" is used when this procedure is selected as "Core Procedures". This precondition is handled as the goal status in constructing the "Procedure" queue. In contrast with the former, "Possible precondition" is used when this procedure is selected for the data transformation, that 


\section{A Knowledge Supporting System for Data Analysis}

is, this is just the precondition in problem solving. A delete-list means the information lost by the procedure, and an add-list means the added one.

The default values in the "Options" are used for the execution of the procedure. It is possible that a user inputs the values appropriately, but a user could not usually input them because of inadequate knowledge about statistics. Thus, the default values are given in advance. If the default value is not suitable for the data, a user can input the appropriate value or stop the execution.

This example is about the procedure "Principal Components Analysis". Its "Best preconditions" is that the data matrix is not square, the dimension is more than two and all the scales are interval. If the dimension is one, this procedure cannot be used as "Core Procedure", but can be used as one to transform.

When the procedure is executed, the information about the scales and the dimensions are lost once, and then the one about scales is newly added as "interval". The term "acc_prop" in the "Options" means calculating an accumulated proportion, and if the value exceeds the limit value described in the "Options", the search process cannot choose this procedure. The default value of the number of principal components is two.

The condition "dim $>=p$ " means that the number of the principal components (=p) must be less than the dimension of the original data.

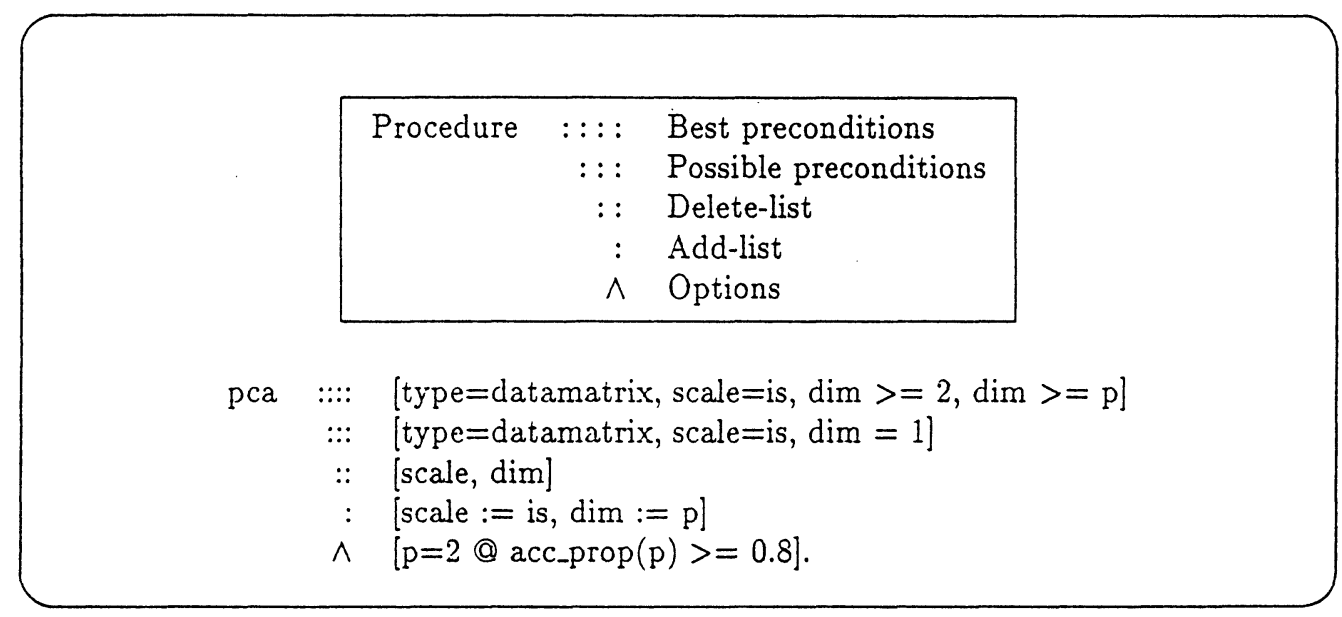

Figure 2: An example of knowledge for construction of a procedure queue (PCA)

Both knowledge bases combine with each other by "Procedure". Each of them is constructed individually. Thus, we can maintain (extend) each of them.

In the practical construction of these knowledge bases, we refer to some literature on data analysis. For example, Kawaguchi $(1973,1978)$ has the table in which some procedures divide into four classes roughly, and each of them divides into two or three whether the 
data are qualitative and/or quantitative. Shiba etal.(1984) have the tree structure of data analysis procedures. Tanaka and Wakimoto(1983) have the flow-chart for the selection of analysis procedures.

\section{How to generate a "Procedure" queue}

Now, we shall explain the algorithm of the system.

\section{Input}

The system requests a user "Data Information" of the observation and "Purpose" for the analysis. He can input them partially with menu-select function by Shimomura et al.(1992). Most of them are handled with intermediate files.

As the system can handle them, it is necessary to interpret most of "Data Information" of the observation in advance according to the form.

Next, the system asks him whether the observation has an external criterion. This operation is done interactively about all the information (knowledge base, "Purpose" and "Data Information" of the observation).

There are three types of choices about an external criterion. If the data have an external criterion, the system will show the features of external criteria as a choice. If not, the system will show a choice "Not match". If a user cannot understand what the question means, he can select "Unknown". Then, the system does not regard the answer "Unknown" as the elements of the retrieval information, and continues the algorithm.

The answer "Unknown" is available for almost all questions.

2. Choose the candidate of "Core Procedure"

According to the result of the previous step, the system retrieves an appropriate "Core Procedure". Candidates are narrowed down, but some candidates may be returned if the system could not lead to the best candidate from the knowledge base, or "Unknown" is included in the retrieval information.

If the number of candidates is more than two, a user must select one of them. Of course, he may not select appropriately. After selection, if the result of the selected "Core Procedure" does not satisfy him, he will be able to go back here, and re-select another candidate. This action is based on the idea of "Generate and Test", which is a basic approach in AI; for example, Shirai and Tsujii(1985) explains it well.

If no candidate is found, the algorithm stops.

\section{Construct a queue of "Procedure"}

The system tries the problem-solving process whose initial status is the "Data Information" of the observation and goal status is the "Best precondition" of the selected "Core Procedure". The searching order depends on the Prolog-system. This step is executed non-deterministically.

If no more queue is available, the algorithm stops. 


\section{Execute and Interpret}

The system executes the queue constructed by the previous step. The data are handled at this stage. Sub-procedures and/or checks of the quantitative conditions described in the knowledge base for construction of "Procedure" queues will be done.

As the result of these procedures, the system may tell a user the necessity of preprocedures, or that the data do not satisfy the quantitative conditions of some "Procedures". Thus, he will go back to the previous steps, and re-select another queue or candidates. If he tried all queues and candidates, it means the system cannot handle the data, and then the algorithm stops.

Interactions among the information are shown in Figure 3.

We cannot estimate the precise size of this system because it consists of three parts which are written with different languages independently. In the inference part (written with Prolog), the number of all the lines of the source files of this system is about 1400 .

USER

SYSTEM

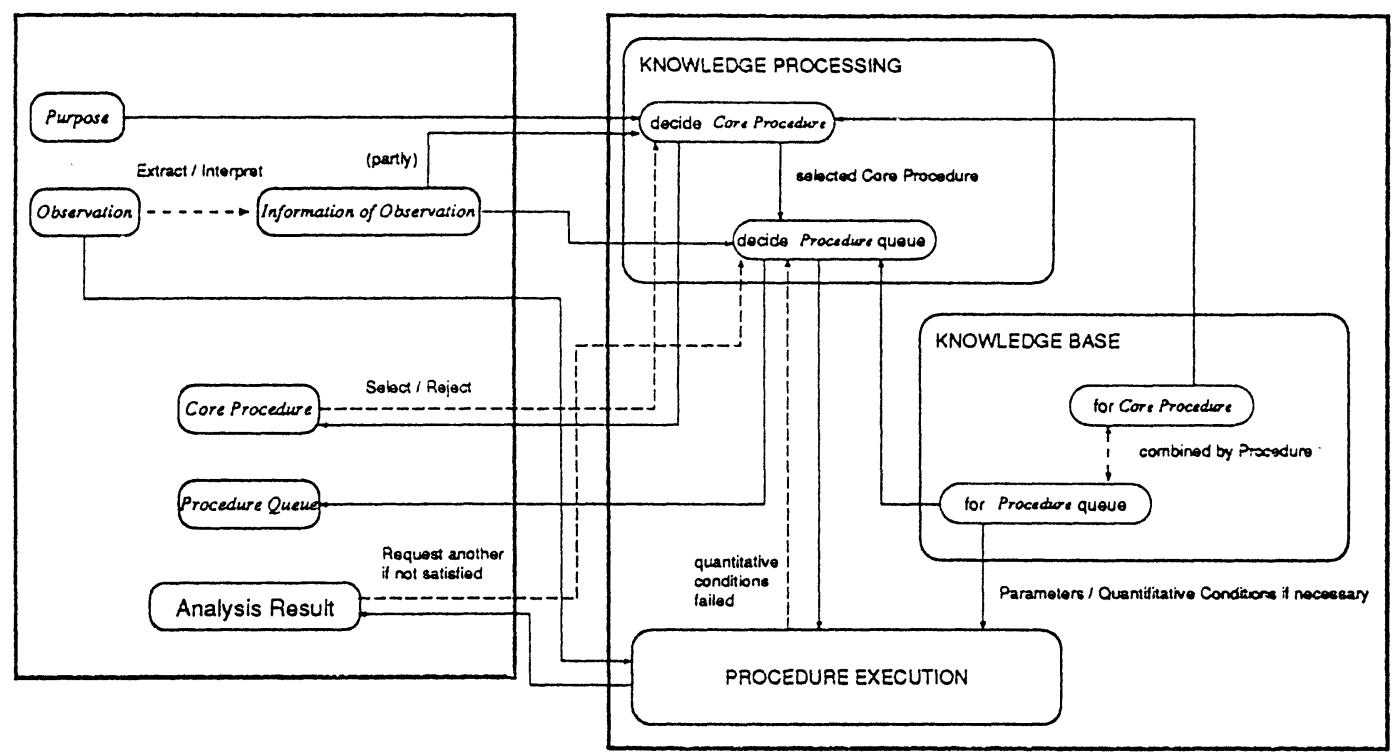

Figure 3: System Overview: Solid arrows mean the flow of information. Dashed arrows mean other actions or requests.

\section{Examples}

The data of the first example is shown in Andrew etal.(1985). Relative weight, fasting plasma glucose, glucose area, Insulin area and SSPG are given on each of the patients, and already divided into three classes. Then, we execute the system in order to confirm 
whether the classification of Class 1 and Class 2 are correct or not. At first, we describe the "Data Information" of the observation in the form that the system can handle. The "Data Information" are shown in Table 1 . Next, we start the system. The process of the execution is shown in the Appendix A. The system asks us the purpose. In some choices here, we select "Classification".

Then, the system searches "Core Procedures" for this purpose, and asks conditions for narrowing down candidates. Here, since we know the data are divided into two groups, we choose "two groups". Thus, the system asks the size of the first group, then we input ' 33 '. Finally, the system tells us "Discriminant Analysis" as the "Core Procedure".

Since the "Data Information" fit the procedure "Discriminant Analysis", transforming procedures are not needed. Then, the procedure is applied directly to the observation. The system cannot find the output procedure appropriately on discriminant analysis, then it suggests a file-output as an output method.

Table 1: The sample data and "Data Information" of the observations about the first example

\begin{tabular}{|r|rrrrr|}
\hline Class & $\begin{array}{c}\text { Relative } \\
\text { weight }\end{array}$ & $\begin{array}{c}\text { Fasting } \\
\text { plasma glucose }\end{array}$ & $\begin{array}{c}\text { Glucose } \\
\text { area }\end{array}$ & $\begin{array}{c}\text { Insulin } \\
\text { area }\end{array}$ & SSPG \\
\hline \hline 1 & 0.92 & 300 & 1468 & 28 & 455 \\
1 & 0.86 & 303 & 1487 & 23 & 327 \\
$\vdots$ & $\vdots$ & $\vdots$ & $\vdots$ & $\vdots$ & $\vdots$ \\
1 & 1.11 & 328 & 1246 & 124 & 442 \\
1 & 0.74 & 346 & 1568 & 15 & 253 \\
& & & & & \\
2 & 0.99 & 98 & 478 & 151 & 122 \\
2 & 1.02 & 88 & 439 & 208 & 244 \\
2 & 0.83 & 103 & 533 & 120 & 135 \\
\hline
\end{tabular}

\footnotetext{
[file=sample36, type=data, size=112, items $=5$, labels=[rel_peight:is, fast_p_glu:is, glu_area:is, ins_area: is, sspg:is] ]
}

The results and plotting of the first and second canonical discriminant variates are shown in Figure 4.

The second example is shown in Table 2. The data is on height, weight, chest, and sitting height of forty male. We want to get some standards from these data. The process of the execution is shown in the Appendix B. The purpose is "Reduction", so discriminant analysis is not a candidate. Thus, the system does not ask whether the data divide into two or not. Since the system cannot find a condition about an external criterion from the knowledge base, the system shows us two candidates "Unknown" and "Not match" about an external criterion. Here we choose "Unknown" because we have already known the data have no external criterion. 
Then, the system cannot select a unique candidate, and shows MDS and PCA as the "Core Procedure". As we must select one of them, we choose PCA here. The procedure PCA has the condition about an accumulated proportion in its knowledge-base. The value for these data is more than 0.8 in the case where the number of principal components is 2 as the default value, so the process continues. If less than 0.8 , other procedures may be selected in "Core Procedure" selection step or "Procedure Queue" selection step.

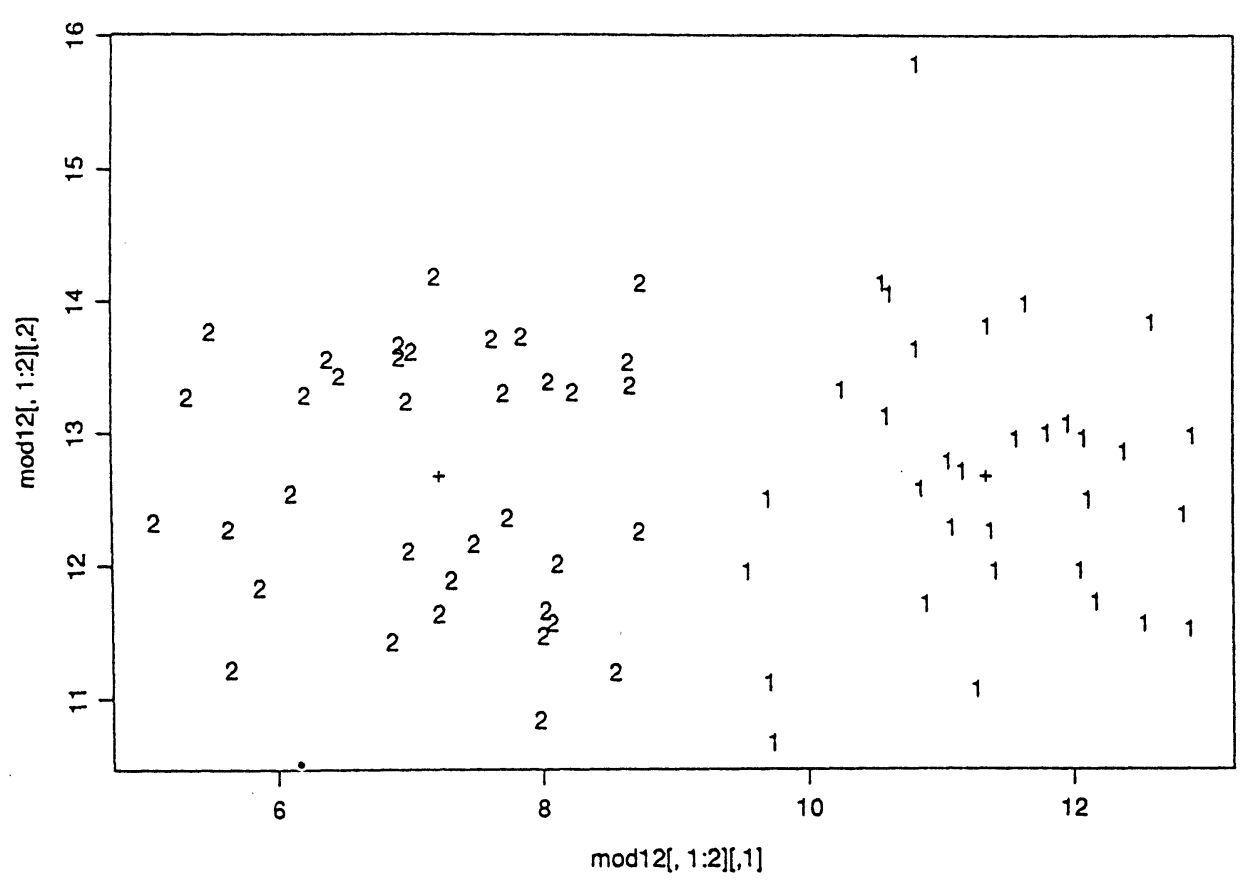

Figure 4: Plotting of the first and second canonical discriminant variates 
Table 2: The sample data of forty male, and "Data Information" of the observations

\begin{tabular}{|r|r|r|r|r|}
\hline ID & Height & Weight & Chest & Sitting Height \\
\hline 1 & 167.5 & 52.5 & 82.0 & 99.5 \\
2 & 172.0 & 62.5 & 88.2 & 99.3 \\
$\vdots$ & $\vdots$ & $\vdots$ & $\vdots$ & $\vdots$ \\
40 & 170.0 & 60.2 & 84.2 & 98.7 \\
\hline
\end{tabular}

[file=sampleA, type=data, size $=40, \operatorname{dim}=4$, labels $=$ [height: is, neight:is, chest:is, sit_height:is]]

\section{Concluding remarks}

In this paper, we investigate the approach of the selection of data analysis procedures by using the concept of knowledge engineering. We offer the system which proposes the queues of the appropriate procedures semi-automatically based on the qualitative information such as a purpose of an analysis and a data structure of observations. We also give some examples in this paper and confirm the efficiency of the function of knowledge supports on statistical softwares.

We still have several problems. First, we must enrich the knowledge. The current version is still in development, and the amount and density of the knowledge are insufficient for a practical analysis. Next, we must investigate whether the more reasonable elements of the knowledge base exist or not. We restrict quantitative judgments to as few as possible, but they are inevitable in data analysis. Prototype and some considerations are necessary as to how much information is required for quantitative judgment and whether the system can handle them or not. Improvement of interface and development of tools in constructing a knowledge base are important topics. The system explained here is not user-friendly now. DINDE and REX have a good interface. We are also constructing the interface.

The software for getting knowledge from statisticians is also required. Recently, Explanation Based Learning (EBL) has attracted attention. It seems to be an efficient approach for many examples about data analysis being accumulated through the system operation by statisticians, and sorting the information from the examples with EBL, and using them for search and inquiries.

\section{REFERENCES}

Andrews et al.(1985) Andrews, D.F. and Herzberg, A.M.(1985). DATA, A Collection of Problems from Many Fields for the Student and Research Worker, Springer-Verlag.

Becker et al.(1988) Becker, R.A., Chambers, J.M. and Wilks, A.R (1988). The New S Language, Wadsworth \& Brook/Cole Advanced Books \& Software, Pacific Grove. 
Furukawa and Mizoguchi(eds.)(1988) Furukawa, K. and Mizoguchi, F.(eds.)(1988). Knowledge Programming, Kyoritsu Shuppan.

Gale(1986) Gale, W.A(ed.) (1986).Artificial Intelligence \& Statistics, Addison-Wesley.

Ishibashi and Takeda(1990) Ishibashi, Y. and Takeda, K.(1990). An expert system for data analysis S/EXP and its effectiveness, J. Japanese Soc. of Computa. Statist., 3, $1,61-66$.

Ishizuka and Kobayashi(eds.)(1991) Ishizuka, M. and Kobayashi, S.(eds.)(1991). Expert System, Maruzen.

Kawaguchi(1973,1978) Kawaguchi, M. $(1973,1978)$. Introduction to Multivariate Analysis (Tahenryo Kaiseki Nyumon, in Japanese) I,II, Morikita Shuppan.

Minami and Mizuta(1990) Minami, H. and Mizuta, M. (1990). An AI Application under a Visual Software Environment, Bulletin of the Faculty of Engineering, 149, 125-133, Hokkaido University.

Minami et al.(1991) Minami, H., Mizuta, M. and Sato, Y.(1991). A knowledge supporting function in data analysis system, in the 59th Annual Meeting of the Japan Statistical Society, 20-22.

Minami and Mizuta(1992) Minami, H. and Mizuta, M.(1992). An expert system on the programs of multi dimensional data analysis, in the 60th Annual Meeting of the Japan Statistical Society, 245-247.

Nakano et al.(1991) Nakano, J., Yamamoto, Y. and Okada, M.(1991). A Knowledge-based Multiple Regression Analysis Surting System, Japanese J. of Applied Statist., 20, 1, 11-23.

Oldford and Peters(1988) Oldford, R.W and Peters, S.C (1988). DINDE: Towards more sophisticated software environments for statistics, SIAM J. Sci. Statist. Comput., 9, 191-211.

Shiba et al.(eds.)(1984) Shiba, Y., Watanabe, H. and Ishizuka, T.(1984). Dictionary for Statistical Terms, (Toukei Yougo Jiten, in Japanese), Shin'yo-sha.

Shimomura et al.(1992) Shimomura, T., Minami, H. and Mizuta, M.(1992). Construction of data analysis environment on $\mathrm{X}$-Window system with knowledge support function, in the 6th Annual Meeting of the Japanese Society of Computational Statistics, 86-87.

Shirai and Tsujii(1985) Shirai, Y. and Tsujii, J.(1985). Artificial Intelligence, Iwanami Information Science 22, Iwanami Shoten.

Tanaka and Wakimoto(1983) Tanaka, Y. and Wakimoto, K.(1983). Multivariate Statistical Analysis Method, (Tahenryo Toukei Kaisekihou, in Japanese), Gendai-Suugakusha.

(Recieved October 1992; Revised November 1993) 


\section{Appendix A. The process of execution in the first sample}

I ?- $\operatorname{sysinit}(\operatorname{Ret}, \mathrm{A})$.

Welcome to our system (in development).

Select your Purpose.

1: reduction

2: structure

3: classification

I: 3 .

Data Information (inner expression):

[file $=$ sample 36 , type $=$ data, size $=69$, dim $=5$,

labels=[rel_w:is,fast_p_glu:is,glu_area:is,ins_area:is,sspg:is]]

Purpose : classification

I found 3 procedures.

Give me more information on the external criterion.

Please Select the Condition for the Data:

1: Unknown

2: Not matching any condition(s)

3: Data : 2 Groups

I: 3 .

Please input the last number of the 1 st group

1: 1

2: 2

3: 3

69: 69

I: 33.

You may give me more information on scales, if not, I must decide the Core procedure.

Information:[purpose=classification,extcrt=group $([(1,33)$, rest $])$, scale=interval $]$

Candidate: discr

Processing :

sample $36 \stackrel{ }{\Rightarrow}=>$ discr $\Rightarrow$ dat 4386

Processing :

dat4386 $=\Rightarrow$ file $==>$ dat 4392

$A=[$ discr,file $]$,

Ret $=$ discr?

yes

1?- 


\section{A Knowledge Supporting System for Data Analysis}

\section{Appendix B. The process of execution in the second sample}

1?- sysinit(Ret,A).

Welcome to our system (in development).

Select your Purpose.

1: reduction

2: structure

3: classification

I: 1 .

Data Information (inner expression):

[file $=$ sample $A$, type $=$ data, size $=40$, dim $=4$, labels=[height:is, weight:is, chest:is,_si__height:is]]

Purpose : reduction

I found 3 procedures.

Give me more information on the external criterion.

Please Select the Condition for the Data:

1: Unknown

2: Not matching any condition(s)

I: 2.

You may give me more information on scales, if not, I must decide the Core procedure.

Information: [purpose=reduction, extcrt $=$ none,scale $=$ interval]

Candidates: [mds,pca]

Which Procedure do you want to set as Core?:

1: mds

2: pca

I: 2.

Processing :

sampleA $=>$ pca $\Rightarrow$ dat4498

Meta-rule check

Check: acc_prop

Rule: acc_prop $>=.8$ is true.

Processing :

dat $4498 \stackrel{\Rightarrow}{=}$ file $==>$ dat 4501

$A=[p c a$, file $]$,

Ret $=$ pca ?

yes 